\title{
Aplicación de arquitecturas peer-to-peer a la distribución de archivos audiovisuales
}

\author{
Por David Fernández-Quijada
}

\begin{abstract}
Resumen: La distribución de archivos audiovisuales de gran tamaño a través de redes electrónicas topa con problemas como la falta de escalabilidad y el coste excesivo. Ante esta situación algunas instituciones y empresas están aplicando arquitecturas de red peer-to-peer que permiten optimizar la transmisión y reducir su coste. Este artículo repasa la naturaleza y características de este modelo, así como algunos de los principales proyectos que ya lo están poniendo en práctica. Analiza las ventajas asociadas a este sistema y la contribución que puede hacer a una distribución eficiente de archivos que acerque el patrimonio audiovisual a una ciudadanía cada vez más formada en la cultura de las imágenes y el sonido.

Palabras clave: Peer-to-peer, Tecnología, Archivos audiovisuales, Televisión, Digitalización.

\section{Title: Peer-to-peer architectures applied to the distribution of audio-} visual archives

Abstract: The distribution of large audiovisual archives through electronic networks has problems like the lack of scalability and an excessive cost. To deal with these obstacles, some institutions and broadcasters are applying peer-to-peer architectures in order to optimize the transmission and to reduce its cost. This article explains this model and its main characteristics, as well as some of the projects which are using it nowadays. It analyses the advantages associated to this system and the contribution it can make for a more efficient distribution of archives. The aim is to make the audiovisual heritage available to a citizenry educated in a culture of images and sound.
\end{abstract}

Keywords: Peer-to-peer, Technology, Audiovisual archives, Television, Digitisation.

Fernández-Quijada, David. “Aplicación de arquitecturas peer-to-peer a la distribución de archivos audiovisuales". En: El profesional de la información, 2008, septiembre-octubre, v. 17, n. 5, pp. 526-531.

DOI: 10.3145/epi.2008.sep.06

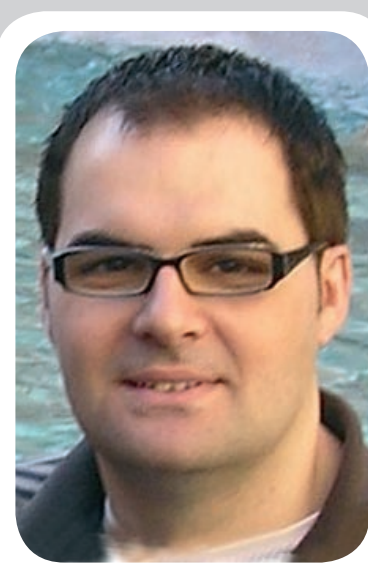

David Fernández-Quijada, licenciado en comunicación audiovisual y doctor por la Universitat Autònoma de Barcelona, en cuyo Departament de Comunicació Audiovisual $i$ de Publicitat ejerce actualmente como profesor ayudante. Es investigador asociado al Grup de Recerca en Imatge, So i Síntesi (Griss) de la misma universidad, donde desarrolla investigaciones sobre tecnologías de la comunicación, economía política de las industrias culturales y el servicio público de radiodifusión.

\section{El valor de la memoria audiovisual digital}

Los archivos audiovisuales constituyen un patrimonio cada vez más importante de las diferentes culturas. Ahí precisamente radica el valor de su adecuada conservación y obviamente de la extensión de sus posibilidades de acceso a científicos, profesionales y ciudadanía en general. En una sociedad que de manera creciente se forma en la cultura de la imagen y del sonido, la función de memoria histórica y cultural de naturaleza audiovisual cobra protagonismo, convirtiéndose en un patrimonio de excepción.

Más allá del valor cultural e histórico, la memoria audiovisual también supone un activo para sus propietarios o gestores desde el punto de vista económico. Véase en nuestro entorno más próximo el caso de $T e$ levisión Española (TVE), la emisora pública del Estado que con motivo de su quincuagésimo aniversario demostró cómo explotar su fondo con la creación del
Canal 50 TVE, exclusivamente alimentado a partir del más del millón de documentos audiovisuales de su archivo. Y ello a pesar de que la cadena no dispuso de una política global de archivo hasta los años 80 (Caldera Serrano, 2003), lo que llevó a la pérdida de valiosas piezas, y de que hoy en día el proceso de digitalización todavía se halle en una fase incipiente, en claro contras-

\section{"La función de memoria histórica y cultural de naturaleza audiovisual cobra protagonismo"}

te con la digitalización ya completada de la división radiofónica del grupo, Radio Nacional de España (RNE). Otros casos de explotación por parte de las instituciones lo constituyen Televisió de Catalunya, que desde 
2004 dispone en internet del servicio bajo demanda 3 a la carta, o, por citar casos relevantes del contexto europeo, el servicio RAI Click de la televisión pública italiana o el británico BBC Motion Gallery.

http://www.rtve.es - Prensa - 31 octubre 2006

http://www.elprofesionaldelainformacion.com/ contenidos/2003/marzo/12.pdf

\section{http://www.tv3.cat/seccio/3alacarta/}

\section{http://www.raiclicktv.it}

\section{http://www.bbcmotiongallery.com}

El proceso de digitalización afecta a todos los estadios de la cadena de valor en la industria de radiodifusión: la producción, la distribución y el consumo de señales audiovisuales que se transmiten a través de ondas electromagnéticas. En muchos casos además "libera la circulación y el uso del contenido de las limitaciones impuestas por el espacio, el tiempo y la especificidad de los antiguos soportes" (Lacroix; Tremblay, 1997, pp. 35-36), lo que a la vez permite su consulta o consumo a través de redes electrónicas como internet. Someramente recordaremos algunos de los motivos que Spence (2002) utiliza para justificar la digitalización de un archivo:

- inminente obsolescencia de la tecnología analógica;

- preservación de la información;

- necesidad de un medio de almacenaje estable;

- recuperación más fácil y rápida;

- aumento en los niveles de acceso;

- mayor seguridad;

- ahorros económicos y de espacio en el almacenaje; y

- generación de ingresos adicionales.

Este proceso representa un esfuerzo notable para los radiodifusores, en especial para aquellos de mayor longevidad y que lógicamente acumulan un fondo más grande. La flexibilidad que otorgan las tecnologías debe aplicarse además inteligentemente para abordar la fase posterior a la conservación, esto es, una difusión que aumente el valor intrínseco con el que ya cuenta el archivo. Y en el caso que nos ocupa esto se traduce en desprenderse del soporte para facilitar y agilizar la transmisión de los contenidos a través de redes electrónicas, ya sea con un modelo de negocio gratuito o de pago.

En el presente artículo se repasan algunos de los principales problemas de la difusión de archivos de naturaleza audiovisual en internet para explicar las so- luciones que para los mismos supone la arquitectura de distribución de archivos peer-to-peer, de la que se examina su funcionamiento y se determinan las ventajas que aporta frente a los modelos de distribución tradicionales para mostrar, en último término, algunas de las principales implementaciones de la misma en dos campos: los archivos audiovisuales y la radiodifusión.

\section{Problemas en la difusión audiovisual en internet}

La distribución de contenidos audiovisuales a través de la Red se puede realizar de dos formas distintas: con descarga previa, de manera que el archivo queda alojado en el dispositivo del usuario, o a través de streaming, es decir, la reproducción se realiza conforme va llegando el material al terminal del usuario, sin necesidad de descargarse completamente. Ambos sistemas presentan dificultades, ya que "la transmisión de contenidos multimedia por internet nació con dos problemas: tamaño y coste. El reto es transmitir una señal a muchos receptores simultáneos y hacerlo sin que los costes crezcan en proporción al tamaño de la audiencia" (Alstrup; Rauhe, 2005, p. 1).

El problema del tamaño se relativiza con el paso del tiempo gracias a dos factores: en primer lugar, un mayor ancho de banda en las conexiones de los usuarios. En segundo, el perfeccionamiento de técnicas de tratamiento de la señal digital como la compresión que permite reducir el tamaño de los archivos a transmitir. Aun así la difusión de archivos de naturaleza audiovisual en internet continúa presentando problemas en cuanto al coste, ya que una gran demanda simultánea de archivos puede bloquear los servidores u obligar a una inversión en la multiplicación de las capacidades de hosting. En el caso del streaming, además, el problema va de la mano de la popularidad, ya que una alta demanda de un contenido implica un aumento de las necesidades de ancho de banda y, por lo tanto, un mayor coste para el distribuidor de ese contenido.

A estos problemas cabría añadir la falta de escalabilidad que aparece con un elevado número de usuarios simultáneos, un factor que también afecta a la robustez del sistema si éste no está bien dimensionado para satisfacer esta demanda concurrente.

\section{Nuevas propuestas en torno a la arquitectura peer-to-peer}

Los dos problemas descritos se relacionan directamente con la propia arquitectura de internet. Ésta se basa en la llamada de una máquina cliente (habitualmente, el ordenador personal de cualquier usuario) a otra que actúa de servidor (los grandes ordenadores dedicados que alojan las aplicaciones y los sitios de 
internet), que envía al cliente la información que ha requerido. Ambas se identifican a través de dírecciones o números Ip, que son únicos y las diferencian del resto de máquinas de la Red. En este esquema cada uno de los dos extremos de la comunicación tiene su función perfectamente definida.

Sin embargo otro tipo de arquitecturas de red como el peer-to-peer ( $\mathrm{P} 2 \mathrm{P})$ pueden salvar los obstáculos que presenta el modelo tradicional. Este concepto hace referencia a un sistema de transmisión de datos en red que basa su funcionamiento en un sistema sin jerarquías donde todas las máquinas son a la vez clientes y servidores. Por ello se le denomina intercambio entre pares o entre iguales. Todos los dispositivos $-\mathrm{y}$ usuarios- integrantes de una red $\mathrm{P} 2 \mathrm{P}$ pueden comunicarse directamente entre sí sin la obligación de circular a través de un servidor, lo que los dota de un mayor grado de autonomía. Este modelo cuenta con la ventaja de ser complementario, y no excluyente, del modelo cliente/ servidor. Su implementación masiva se da sobre redes Ip, constituyéndose en redes virtuales que funcionan sobre la infraestructura técnica de internet pero singularizadas porque la búsqueda de la información no se realiza en función de la ubicación del recurso sino de su descripción (Pérez Subías, 2003).

\section{"Los integrantes de una red P2P son más autónomos ya que pueden comunicarse directamente entre sí sin tener que pasar por un servidor"}

La identificación a través de direcciones Ip es inestable ya que los equipos cliente se conectan a través de direcciones Ip dinámicas asignadas al momento por el proveedor de acceso (internet services provider, ISP) para cada conexión. El proveedor ISP dispone de un número de direcciones Ip que las va asignando automáticamente a sus clientes cada vez que conectan. La arquitectura P2P identifica en cambio cada dispositivo que se conecta a través de un sistema dinámico propio de asignación de identificadores, pasando por encima de la jerarquía Ip. Más allá del terminal desde el que se conecta el usuario, el sistema permite reconocerle al instante gracias a este identificador único.

El proyecto Open $P 2 P$ mantiene el más extenso directorio de aplicaciones P2P de la Red. El estado embrionario de muchas de estas aplicaciones y su heterogeneidad no han permitido hasta el momento el establecimiento de una tipología que delimite fielmente las diferentes aplicaciones. Uno de los responsables del proyecto, Tim O'Reilly (citado por Sims, 2000), propuso una clasificación con tres grandes tipos de aplicaciones:

- mensajería instantánea (todas las que comparten contenidos entre usuarios),

- grupos de trabajo (trabajo simultáneo en red desde diferentes espacios) y,

- computación distribuida (utilización de recursos informáticos remotos para determinados procesos).

Esta clasificación adolecía no obstante de falta de consistencia y dejaba muchas aplicaciones huérfanas de una categoría matriz. Otras categorizaciones han partido de una visión más tecnocéntrica, privilegiando una mirada desde el conjunto del avance tecnológico más que desde la propia aplicación o el usuario (Kant; Iyer; Tewari, 2002), sobre la base de criterios como el grado de descentralización del almacenamiento o el control sobre el recurso.

\section{http://www.openp2p.com}

La perspectiva predominante actualmente parte del punto de vista técnico para distinguir hasta tres tipos de aplicaciones peer-to-peer:

- Primera generación (centralizada): los pares o nodos se conectan directamente entre sí pero precisan de un servidor central que les permita la comunicación. En él se alojan los archivos con los metadatos que ponen en contacto las demandas de los usuarios con los contenidos disponibles, a través de la aplicación P2P específica o mediante interfaces web.

- Segunda generación (descentralizada): los pares se comunican entre sí a través de las redes y sin ningún recurso central, de manera que las búsquedas se realizan enteramente a través de la propia red $\mathrm{P} 2 \mathrm{P}$.

- Tercera generación (híbrida): son una mezcla de las dos generaciones precedentes, en lo que se podría llamar un entorno descentralizado controlado. Hay varios "supernodos" a los que se conectan las máquinas de los usuarios y que hacen las funciones de servidor centralizado que pone en contacto a las máquinas. Los archivos permanecen descentralizados.

Más allá de su teorización, los sistemas peer-topeer vienen a resolver los principales problemas identificados en la difusión de archivos audiovisuales en la Red:

- Falta de escalabilidad: éste había sido tradicionalmente el gran problema de los sistemas de difusión streaming en internet: al aumentar la demanda simultánea de archivos crecían proporcionalmente las necesidades de ancho de banda y recursos de hardware. Los sistemas P2P utilizan en cambio recursos instalados 
pero infrautilizados situados en la periferia de internet, en sus usuarios. Entre los más importantes de estos recursos cabe mencionar el ancho de banda, el espacio de almacenaje en disco o la capacidad de procesamiento de las máquinas.

\section{"Los sistemas P2P son escalables, pues cuando hace falta utilizan recursos ya instalados pero infrautilizados de los usuarios"}

- El alto coste: la fórmula P2P se basa en añadir al conjunto del sistema recursos ya instalados y poco utilizados situados en los entornos locales de los propios usuarios, liberando de esta manera a la organización distribuidora del contenido de gran parte de los costes. La propia digitalización ya supone una importante reducción de inversión para los radiodifusores una vez descontado el desembolso que en algunos casos puede llegar a tener el proceso en sí. La utilización de los recursos de los propios usuarios implica una nueva disminución de los costes que para las organizaciones supone la distribución de contenidos en internet. De esta manera se traspasa al usuario la mayor parte del coste de implantación, gestión y mantenimiento del servicio.

- Falta de robustez: la mayor parte se basa en la multiplicación de los nodos que suministran contenidos. El sistema ofrece más recursos y más fuentes para éstos, por lo que la disponibilidad de información es mayor, proporcionando a la vez la capacidad de transmisión necesaria. El fin de la centralización de los sistemas permite que éstos sean también más resistentes ante posibles ataques externos. De esta manera el contenido propiedad de un emisor o una institución es replicado en diferentes máquinas del sistema que pueden proveer al siguiente usuario que pida acceso al mismo contenido, ya sea de manera complementaria o alternativa.

\subsection{El modelo Birth}

La arquitectura peer-to-peer no es sólo una concepción teórica con respecto a la distribución audiovisual en internet sino que existen proyectos que la están llevando a cabo. En el entorno documental, quizá uno de los más importantes sea Birth (Building of an Interactive Research and Delivery Network for Television Heritage) financiado por el programa Media Plus de la Comisión Europea. Siguiendo la tipología descrita por Codina (2003), puede ser considerado como un banco audiovisual. Su objetivo es la creación de una plataforma de intercambio de material audiovisual digitalizado (Hecht; O'Dwyer; Oomen; Scharinger, 2004), accesible desde una interfaz de amplia implantación como la Web. Iniciado en 2003, se centra en brindar mayores oportunidades de acceso a los archivos audiovisuales de las instituciones participantes.

\section{http://www.birth-of-tv.org}

Para los emisores supone nuevas posibilidades de difusión y, si el modelo de negocio lo permite, de generación de ingresos a partir del patrimonio audiovisual que ya poseen. Los documentos audiovisuales disponibles pertenecen a los archivos de emisoras públicas como la British Broadcasting Corporation (BBC), la austríaca Österreichischer Rundfunk (ORF), la belga Radio Télévision Belgique Française (RTBF) o la alemana Südwestrundfunk (SWR), así como al centro holandés Nederlands Instituut voor Beeld en Geluid. Junto a estos poseedores de contenidos, el peso técnico del proyecto recae sobre el instituto austríaco Joanneum Research y la compañía holandesa Noterik Multimedia. Por parte del mundo académico está presente la Hagenberg University of Applied Science, Linz, Austria.

Los archivos se pueden previsualizar en diversas resoluciones (por ejemplo, la $R T B F$ ofrece en Windows Media dos opciones de vídeo a 320×240 y a 192×144 píxeles), aunque la disponibilidad de esta opción depende de las condiciones con que cada operador ha cedido los vídeos, así como de las decisiones previas sobre resolución y calidad adoptadas por las instituciones en su proceso de digitalización. Con el objetivo de optimizar su transmisión los archivos se distribuyen a los usuarios a través de una plataforma peer-to-peer que permite poner en práctica las concepciones teóricas expuestas en el apartado anterior.

La posibilidad de acceder a este material en línea es uno de los aspectos más difíciles de gestionar, dada la heterogeneidad de las diferentes leyes nacionales a las que se deben los diferentes operadores implicados en el proyecto. La falta de armonización legislativa a nivel europeo es una de las principales dificultades en el acceso a este material, a pesar de la consciencia que tiene la propia Comisión Europea sobre la importancia del patrimonio cultural para el desarrollo social y económico en la era digital. En ocasiones ni tan siquiera se sabe con certeza a quién pertenecen los derechos o con quién se deben negociar. Muchos de los acontecimientos guardados en esos archivos proceden de una época en la que la negociación sobre derechos apenas se había iniciado.

Una de las ventajas que ofrece Birth es el uso de metadatos para identificar la información, a partir de los parámetros de la Dublin core initiative y del están- 
dar mpeg7. Esta información comprende fundamentalmente archivos audiovisuales pero también fotografías y textos que se integran en un entorno típicamente multimedia. El sistema emplea taxonomías referidas a los géneros audiovisuales, clasificación geográfica y palabras clave, e incluso para algunos archivos dispone de story-board, muy útil por ejemplo para los estudiosos de la narrativa audiovisual. Las palabras clave se basan en el tesaurus del International Press Telecommunications Council (IPTC), al que se añaden aportaciones propias de los integrantes de Birth (Hecht, 2004), que actualmente se halla en plena migración a otro proyecto mucho más ambicioso que amplía el número de participantes (se incorpora por ejemplo Televisió de Catalunya) llamado Video Active, que mantiene la misma filosofía y la plataforma de distribución peer-to-peer. Tanto éste como Birth se conciben además como medio de estudio tanto de la televisión como de la propia historia europea a través de sus imágenes.

http://www.videoactive.eu

\subsection{El peer-to-peer como broadcast}

Más allá de su utilización estricta para la explotación de archivos audiovisuales, en los últimos meses varios radiodifusores están probando la tecnología P2P como una alternativa en su difusión webcasting, es decir, a través de internet. En España TVE emite sus canales temáticos Canal 24 Horas y Docu TVE a través de P2P, así como la señal de Radio Clásica y Radio Exterior de España. Los derechos de los contenidos que emiten estos canales pertenecen a TVE, ya que la dificultad de establecer fronteras geográficas en internet limita la explotación de contenidos de los que no se disponga de derechos a nivel mundial. Nuevamente aparece en este caso la dificultad de gestionar los aspectos legales, que representan mayores problemas que los meramente técnicos.

\section{http://www.rtve.es/ip2prtvel}

El servicio de TVE se basa en GridCasting, una tecnología propia desarrollada por la compañía danesa $O c$ toshape sobre una arquitectura peer-to-peer. Además de RTVE la utilizan los operadores públicos portugués y esloveno, Rádio e Televisão de Portugal (RTP) y RTV Slovenija, el servicio internacional alemán Deutsche Welle y el canal de la Comisión Europea, entre otros. En todos estos casos la arquitectura P2P se utiliza para un servicio en streaming, ya que la transmisión de estos canales es continua.

En el caso del canal público español los metadatos asociados a las emisiones nos informan de que Docu TVE emite a 384x288 píxeles, con el códec de video Windows Media 9, el códec de audio Windows Media 9.1 y sonido a $64 \mathrm{Kbps}$ de bit-rate y a una frecuencia de $48 \mathrm{KHz}$ en estéreo. En total el canal utiliza 400
Kbps para transmitir su señal. En el caso de Canal 24 Horas, con un mayor bit-rate de transmisión, el sonido se codifica a $128 \mathrm{Kbps}$ para un total de $450 \mathrm{Kbps}$. Estos datos se podrían considerar típicos en el contexto actual de internet. El modelo de RTVE no difiere demasiado del de otros canales europeos, como el operador público nacional danés Danmarks Radio, que cambia la solución tecnológica de Octoshape por el Intelligent Content Delivery de la compañía RawFlow (Jevnaker, 2006), basado en la misma arquitectura peer-to-peer.

Otro experimento destacable es el de Flexible $T V$, una aplicación desarrollada para permitir el visionado de los contenidos de la $B B C$ desde un ordenador personal. Lo novedoso del proyecto reside en el modo de distribución de estos contenidos, ya que el sistema se ha diseñado bajo una arquitectura peer-to-peer específica, BitTorrent, masivamente utilizada para el intercambio de archivos. A partir de una simple copia colocada en el servidor de la cadena, el contenido se va replicando en los dispositivos de los usuarios que se bajan el programa y así los siguientes usuarios tienen más fuentes desde las que obtener el mismo contenido. De hecho esto produce "un círculo virtuoso: a mayor popularidad del programa, mayor número de copias en la Red y mayor facilidad para obtener el contenido" (Pesce, 2004).

\section{"La comunicación peer-to-peer valoriza nuevos modelos de difusión de la información, facilitando el acceso a los archivos digitales"}

\section{Conclusiones}

A través de diferentes modelos basados en P2P, los operadores audiovisuales tradicionales superan el modelo broadcasting en el que un emisor distribuye un contenido simultáneamente a múltiples receptores. Aplicado en internet, este modelo no resulta sostenible por su alto coste. Con la aplicación de la arquitectura peer-to-peer, la idea que emerge es la de convertir una red vertical (jerárquica) en otra de tipo horizontal en la que todos sus nodos sean simétricos, es decir, en un mismo nivel de importancia, ya que todos ellos aportan recursos y contenidos al conjunto del sistema (Fernández-Quijada, 2005).

La comunicación peer-to-peer valoriza nuevos modelos de diseminación de la información, facilitando el acceso a los contenidos de los radiodifusores y, entre éstos, a los de sus archivos digitales. Permite afrontar de manera radical la dificultad económica que supone la puesta a disposición de todo este inmenso repositorio 
de alto valor cultural a las diferentes comunidades de usuarios. En el horizonte próximo, el progreso en los sistemas de estandarización y de descripción de metadatos promete además nuevas mejoras.

No obstante, el auténtico valor de la digitalización se muestra en aplicaciones como el peer-to-peer, que permiten optimizar la naturaleza digital de los datos para una mayor difusión, lo que repercute en una aumento en la facilidad de acceso para los usuarios y del servicio a éstos por parte de las instituciones responsables de los archivos. Por otra parte, en un plano más conceptual, este tipo de sistemas aumentan el valor social tanto de los archivos institucionales como de los servicios de radiodifusión al permitir un uso más intensivo de los mismos.

\section{"Se necesita simplificar los sistemas de gestión de derechos si realmente se quiere hacer un uso social y económico del inmenso patrimonio audiovisual"}

Los progresos técnicos que permite esta arquitectura de difusión aún se deben complementar con otros que quedan pendientes, como los aspectos legales. La regulación de los derechos de propiedad intelectual y de los propios derechos sobre los contenidos generados por los operadores televisivos sigue siendo dificultosa para los operadores, que no pueden apostar por una amplia difusión de sus archivos sin un sistema que garantice su integridad y evite reclamaciones. Sin duda, a este nivel parece necesaria una simplificación de los sistemas de gestión de derechos y una mayor implicación desde el nivel normativo si realmente se quiere hacer un uso social y económico del inmenso patrimonio audiovisual generado.

\section{Bibliografía}

Alstrup, Stephen; Rauhe, Theis. "Octoshape: a new technology for large- scale streaming over the internet". En: EBU technical review, 2005, n. 303 Consultado en: 28-07-08.

http://www.ebu.ch/en/technical/trev/trev_303-octoshape.pdf

Caldera-Serrano, Jorge. "La documentación audiovisual en las empresas televisivas”. En: Biblios, 2003, n. 15, pp. 3-11.

Codina, Lluís. "El nou sector emergent dels bancs audiovisuals en el world wide web". En: Quaderns del CAC, 2003, n. 15, pp. 41-53.

Fernández-Quijada, David. "An optimized architecture for content dissemination: peer-to-peer". En: IASA journal, 2005, n. 26, pp. 44-52.

Haefner, Albrecht. "Renaissance in archiving: the present upheaval in audiovisual archives: evolution towards multimedia archiving?". En: SA archives journal, 2001/2002, n. 42, pp. 7-14.

Hecht, Alexander. "The Birth project". En: ViewFinder, 2004, n. 57, pp. 16-17.

Hecht, Alexander; O'Dwyer, Andrew; Oomen, Johan; Scharinger, Florian. "Birth: building an interactive research and delivery network for television heritage”. En: Proceedings of Ichim digital culture and heritage, 2004. Consultado en: 20-07-08.

http://www.ichim.org/ichim04/contenu/PDF/2280_Oomen.pdf

Jevnaker, Ingjerd-Straand. "RawFlow: using P2P to create virtual 'multicasts".. En: EBU technical review, 2006, n. 308. Consultado en: 10-07-08.

http://www.ebu.ch/en/technical/trev/trev_308-rawflow.pdf

Kant, Krishna; Iyer, Ravishankar K.; Tewari, Vijay. "A framework for classifying peer-to-peer technologies". En: Proceedings of the Second IEEE/ACM international symposium on cluster computing and the grid, 2002, pp. 368-375.

Lacroix, Jean-Guy; Tremblay, Gaëtan. "The 'information society' and cultural industries theory". En: Current sociology, 1997, v. 45, n. 4, pp. 1-154.

Pérez-Subías, Miguel. "Redes P2P una nueva forma de almacenar y acceder a la información”. En: Bit, 2003, n. 141, pp. 28-30.

Pesce, Mark. "F*ck big media: rolling your own network". En: Hyperreal, 2004. Consultado en: 10-07-08.

http://www.hyperreal.org/ mpescelfbm.html

Sims, David. "P2P directory”. En: Open P2P, O’Reilly \& Associates, Santa Clara. Consultado en: 20-06-08.

http://www.openp2p.com/pub/a/p2p/2000/10/20/directory.html

Spence, John. "Dams \& digitization preparedness". En: IASA journal, 2002, n. 20 .

David Fernández-Quijada, Departament de Comunicació Audiovisual i de Publicitat, Universitat Autònoma de Barcelona.

david.fernandez@uab.es

\section{Suscripción EPI sólo online}

Pensando sobre todo en los posibles suscriptores latinoamericanos, ya no es obligatorio pagar la suscripción impresa de EPI para acceder a la online.

EPI se ofrece a instituciones en suscripción "sólo online" a un precio considerablemente más reducido (85 euros/año), puesto que en esta modalidad no hay que cubrir los gastos de imprenta ni de correo postal. 
